\title{
Tests for the Expansion of the Universe
}

\section{Martín López-Corredoira*}

Instituto de Astrofísica de Canarias, E-38205 La Laguna (Tenerife), Spain

Universidad de La Laguna, Dept. Astrofísica, E-38206 La Laguna (Tenerife), Spain

E-mail: martinlc@iac.es

\begin{abstract}
Almost all cosmologists accept nowadays that the redshift of the galaxies is due to the expansion of the Universe (cosmological redshift), plus some Doppler effect of peculiar motions, but can we be sure of this fact by means of some other independent cosmological test? Here I will review some recent tests: CMBR temperature versus redshift, time dilation, the Hubble diagram, the Tolman or surface brightness test, the angular size test, the UV surface brightness limit and the Alcock-Paczyński test. Some tests favour expansion and others favour a static Universe. Almost all the cosmological tests are susceptible to the evolution of galaxies and/or other effects. Tolman or angular size tests need to assume very strong evolution of galaxy sizes to fit the data with the standard cosmology, whereas the Alcock-Paczynski test, an evaluation of the ratio of observed angular size to radial/redshift size, is independent of it.
\end{abstract}

Frontiers of Fundamental Physics 14

15-18 July 2014

Aix Marseille University (AMU) Saint-Charles Campus, Marseille, France

* Speaker. 


\section{Does redshift mean expansion?}

Lemaitre[1] in 1927 and later Hubble[2] in 1929 established the redshift (z)-apparent magnitude relation of the galaxies, which gave an observational hint that the Universe is expanding. Hubble was cautious in suggesting this interpretation, but succeeding generations of cosmologists became pretty sure that the redshift of the galaxies following Hubble's law is a definitive proof of expansion. There were alternative explanations for the redshifts: for instance, "tired light" scenarios[3][4](Sect. 2.1), in which it is assumed the photon loses energy due to some unknown process of photon-matter or photon-photon interaction when it travels some distance. This idea had two main potential problems[3]: the usual scatterings would produce blurring in the galaxies and a frequency-dependent redshift, neither of which is observed, but are solved with exotic nonstandard models of scattering[4](Sect. 2.1). In any case, owing mainly to the absence of a good theory based on standard physics explaining the possible phenomenological fact of these alternative proposals, and given that general relativity provided an explanation for the cosmological expansion while alternative proposals were not supported by any well-known orthodox theory, the expansion hypothesis was preferred and alternative approaches were doomed to be forgotten.

\section{Observational tests for the expansion of the Universe}

Apart from the actual redshift of the galaxies, there are different tests to verify whether the Universe is expanding or static:

1. Microwave Background temperature as a function of redshift.

The Cosmic Microwave Background Radiation (CMBR) temperature can be detected indirectly at high redshift if suitable absorption lines can be found in high redshift objects. Hot Big Bang cosmology predicts that the temperature of the CMBR required to excite these lines is higher than at $z=0$ by a factor $(1+z)$.

The CMBR temperature measured from the rotational excitation of some molecules as a function of redshift $[5,6]$ was quite successful in proving the expansion: the results of Noterdaeme et al.[6] with the exact expected dependence of $T=T_{0}(1+z)$ are impressive. Nonetheless, there are other results that disagree with this dependence[7, 8]. The discrepancy might be due to a dependence on collisional excitation[5] or bias due to unresolved structure[8].

2. Time dilation test.

Clocks observed by us at high redshifts will appear to keep time at a rate $(1+z)$ times slower when there is expansion. By using sources of known constant intrinsic periodicity, we would expect their light curves to be stretched in the time axis by a factor $(1+z)$.

Time dilation tests in Type Ia supernovae (SNIa) look like one of the most successful tests in favour of the expansion of the universe $[9,10]$, but there are still some problems in their interpretation. The fact that SNIa light curves are narrower when redder[11] is an inconvenience for a clean test free from selection effects. Other selection effects and the possible compatibility of the results with a wider range of cosmological models, including static ones, 
have also been pointed out[12, 13][4](Secc. 2)[14](Secc. 7.8). Moreover, neither gammaray bursts (GRBs)[15] nor Quasi Stellar Objects (QSOs)[16] present time dilation, which is puzzling.

\section{Hubble diagram.}

It has been known for many decades that an apparent magnitude (taking into account $\mathrm{K}$ corrections) vs. distance diagram for elliptical galaxies in clusters fits better a static rather than an expanding Universe[17]. This disagreement could, however, be solved by an increase of luminosity at higher redshift due to the evolution of galaxies.

For SNIa[18] or GRBs[19], for which it is supposed there is no evolution, the standard model works, provided that an ad hoc dark energy constant is included. Nonetheless, a static Universe may also fit those data[20, 21].

4. The Tolman surface brightness test.

Hubble and Tolman[22] proposed the so-called Tolman test based on the measurement of the surface brightness. A galaxy at redshift $z$ varies in surface brightness proportionally to $(1+z)^{-n}$ with $n=4$ for expansion and $n=1$ for the static case.

Lubin \& Sandage[23] claimed in 2001 to have definitive proof of the expansion of the Universe using the Tolman test up to $z=0.9$. However, their claim, rather than being a Tolman test, was that the evolution of galaxies can explain the difference between the results of the Tolman test and their preferred model, which includes expansion. Lerner[24] observed that Lubin \& Sandage used a very involved evolutionary k-correction scheme, with many adjustable assumptions and parameters to correct observed high- $z$ surface brightness. Crawford[13] also pointed out that Lubin \& Sandage performed a wrong analysis to exclude the static solution, mixing Big Bang and tired-light models.

Furthermore, other more recent Tolman tests[24, 13, 25], some of them up to redshifts of $\sim$ 5 and with different wavelength filters so that no K-corrections are necessary, favour a static Universe without the need for galaxy evolution.

5. Angular size vs. redshift test.

The angular size $(\theta)$ of a galaxy with a given linear size is very different if we assume the standard model with expansion or a static Universe. Tests were made by several authors[17, 26, 20], and all of them, either in the radio, near infrared or visible, show, over a range of up to redshift 3, a dependence $\theta \sim z^{-1}$, a static Euclidean effect over all scales. This result cannot be reconciled with the standard cosmological model unless we assume a strong evolution of galactic radii which coincidentaly compensates the difference: galaxies with the same luminosity should be six times smaller at $z=3.2$ than at $z=0$ [20]. Neither the hypothesis that galaxies which formed earlier have much higher densities nor their luminosity evolution, merger ratio, or massive outflows due to a quasar feedback mechanism are enough to justify such a strong size evolution[20]; also, the velocity dispersion would be much higher than observed[20]. A static Universe is fitted without any ad hoc element. However, we must be cautious with this interpretation, because of the uncertainty of the galaxy size evolution. 
6. UV surface brightness test.

Lerner[24] proposed a test of the evolution hypothesis that is also useful in the present case. There is a limit on the ultraviolet surface brightness (UV SB) of a galaxy because, when the surface density of hot bright stars and thus supernovae increases, large amounts of dust are produced to absorb all the UV except that from a thin layer. Further increase in the surface density of hot bright stars beyond a given point just produces more dust and a thinner surface layer, not an increase in UV SB. Based on this principle, there should be a maximum $\mathrm{UV}$ (at-rest) SB independent of redshift. This was analysed at high redshift[24, 20] and the result is that the intrinsic UV SB would be prohibitively lower(=much brighter) than 18.5 $\operatorname{mag}_{A B} / \operatorname{arcsec}^{2}$ with the evolution required for the standard model to be compatible with the Tolman or angular size tests. For a static model, however, it would be within the normally expected range. Lerner[24] also argues why alternative explanations (lower production of dust at high redshift, winds or other scenarios) are inconsistent. Nonetheless, Lerner's hypothesis of a maximum UV SB might be incorrect, so this should be further explored before reaching definitive conclusions about this test.

7. Alcock-Paczyński test.

Given a distribution of objects with spherical symmetry, with a radius along the line of sight $s_{\|}=\Delta z \frac{d d_{\mathrm{com}}(z)}{d z}$ and a radius perpendicular to the line of sight $s_{\perp}=\Delta \theta(1+z)^{m} d_{\mathrm{ang}}(z)(m=1$ with expansion, $m=0$ for static), the ratio: $y \equiv \frac{\Delta z}{z \Delta \theta} \frac{s_{\perp}}{s_{\|}}$depends on the cosmological comoving distance $\left(d_{\text {com }}(z)\right)$ and the angular distance $\left(d_{\text {ang }}(z)\right)$ and is independent of the evolution of galaxies, but it also depends on the redshift distortions produced by the peculiar velocities of gravitational infall[27].

López-Corredoira[27] measured $y(z)$ by means of the analysis of the anisotropic correlation function of sources in several surveys, using a technique to disentangle the dynamic and geometric distortions, and also took other values available from the literature. From six different cosmological models (concordance $\Lambda \mathrm{CDM}$, Einstein-de Sitter, open-Friedman Cosmology without dark energy, flat quasi-steady state cosmology, a static universe with a linear Hubble law, and a static universe with tired-light redshift), only two of them fit the data of the Alcock \& Paczyński's test: concordance $\Lambda \mathrm{CDM}$ and static universe with tired-light redshift; whereas the rest were excluded at a $>95 \%$ confidence level. Analyses with further data using Baryonic Acoustic Oscillations (BAO) improve the test and give us a more accurate constraint, but do not exclude neither the static case nor expansion yet[28].

\section{Conclusions}

Table 1 summarizes the analysis of this paper. Apparently, there is no winner yet. The first two tests favour expansion, whereas the following four tests get a less ad hoc fit with the static solution, although this is insufficient to reject expansion. Most of the cosmological tests are entangled with the evolution of galaxies and/or other effects. Tolman or angular size tests need to assume a very strong evolution of galaxy sizes to fit the data with the standard cosmology, whereas the AlcockPaczynski test is independent of the evolution of galaxies. 
Table 1: Cosmological tests.

\begin{tabular}{|c|c|c|}
\hline Test & Expansion & Static \\
\hline$T_{\mathrm{CMBR}}(z)$ & Good fit & $\begin{array}{l}\text { Excess temperature at high } \mathrm{z} \\
\text { due to collisional excitation or } \\
\text { due to unresolved structure. }\end{array}$ \\
\hline Time dilation & $\begin{array}{l}\text { Good fit for SNIa. } \\
\text { Unexplained absence of time } \\
\text { dilation for QSOs and GRBs. }\end{array}$ & $\begin{array}{l}\text { Selection effects, or ad hoc } \\
\text { modification of the theory or } \\
\text { the zero point calibration, or } \\
\text { evolution of SNIa periods. }\end{array}$ \\
\hline Hubble diagram & $\begin{array}{l}\text { Requires introduction of dark } \\
\text { energy and/or evolution. }\end{array}$ & $\begin{array}{l}\text { Good fit for galaxies. Good } \\
\text { fit for SNIa with some models. }\end{array}$ \\
\hline Tolman (SB) & Requires strong SB evolution. & Good fit \\
\hline Angular size & $\begin{array}{l}\text { Requires too strong } \\
\text { evolution of angular sizes. }\end{array}$ & Good fit \\
\hline UV SB limit & Too high UV SB at high $\mathrm{z}$ & Within the constraints \\
\hline Alcock-Paczyński & Good fit & Good fit for tired light \\
\hline
\end{tabular}

\section{References}

[1] G. Lemaître, Un Univers homogène de masse constante et de rayon croissant rendant compte de la vitesse radiale des nébuleuses extra-galactiques, Annales de la Société Scientifique de Bruxelles A47 (1927), 49.

[2] E. P. Hubble, A Relation between Distance and Radial Velocity among Extra-Galactic Nebulae, Proc. US Nat. Acad. Sci. 15 (1929) 168.

[3] J. V. Narlikar, Non-cosmological redshifts, Space Science Reviews 50 (1989) 523.

[4] M. López-Corredoira, Observational Cosmology: caveats and open questions in the standard model, in Recent Research Developments in Astronomy and Astrophysics I, S. G. Pandalai, Ed., Research Signpost, Kerala, p. 561 (2003).

[5] P. Molaro, S. A. Levshakov, M. Dessauges-Zavadsky, S. D’Odorico, The cosmic microwave background radiation temperature at $z_{a b s}=3.025$ toward QSO 0347-3819, Astron. Astrophys. 381 (2002) L64.

[6] P. Noterdaeme, P. Petitjean, R. Srianand, C. Ledoux, S. López, The evolution of the cosmic microwave background temperature. Measurements of TCMB at high redshift from carbon monoxide excitation, Astron. Astrophys. 526 (2011) L7.

[7] J. Krelowski, G. Galazutdinov, P. Gnacinski, CN rotational excitation, Astron. Nachrichten 333 (2012) 627.

[8] M. Sato, M. J. Reid, K. M. Menten, C. L. Carilli, On Measuring the Cosmic Microwave Background Temperature at Redshift 0.89 Astrophys. J. 764 (2013) 132.

[9] G. Goldhaber, D. E. Groom, A. Kim, et al., Timescale Stretch Parameterization of Type Ia Supernova B-Band Light Curves, Astrophys. J. 558 (2001) 359. 
[10] S. Blondin, T. M. Davis, K. Krisciunas, et al., Time Dilation in Type Ia Supernova Spectra at High Redshift, Astrophys. J. 682 (2008) 724.

[11] S. Nobili, A. Goobar, The colour-lightcurve shape relation of type Ia supernovae and the reddening law, Astron. Astrophys. 487 (2008) 19.

[12] S. P. Leaning, New Analysis of Observed High Redshift Supernovae Data Show that A Majority Of SNla Decay Lightcurves can be Shown to Favourably Compare with a non Dilated Restframe Template, in 1st Crisis in Cosmology Conference (AIP Conf. Ser. 822(1)), E. J. Lerner, J. B. Almeida, Eds., AIP, Melville, p. 48 (2006).

[13] D. Crawford, Observational Evidence Favors a Static Universe (Part I), J. Cosmology 13 (2011) 3875.

[14] P. A. LaViolette, Subquantum kinetics: The Alchemy of Creation, 4th ed., Starlane Publ., Niskayana (NY, US) (2012).

[15] D. Crawford, No Evidence of Time Dilation in Gamma-Ray Burst Data (2009), arXiv:0901.4169.

[16] M. R. S. Hawkins, On time dilation in quasar light curves, Mon. Not. Roy. Astron. Soc. 405 (2010) 1940.

[17] P. A. LaViolette, Is the universe really expanding?, Astrophys. J. 301 (1986) 544.

[18] M. Kowalski, D. Rubin, G. Aldering, et al., Improved Cosmological Constraints from New, Old, and Combined Supernova Data Sets, Astrophys. J. 686 (2008) 749.

[19] H. Wei, Observational constraints on cosmological models with the updated long gamma-ray bursts, J. Cosm. Astropart. Phys. 8 (2010) 20.

[20] M. López-Corredoira, Angular-size test on the expansion of the Universe, Int. J. Mod. Phys. D 19 (2010) 245 .

[21] L. A. Marosi, Hubble Diagram Test of Expanding and Static Cosmological Models: The Case for a Slowly Expanding Flat Universe, Advances in Astronomy 2013 (2013), id. 917104.

[22] E. P. Hubble, and R. C. Tolman, Two Methods of Investigating the Nature of the Nebular Redshift, Astrophys. J. 82 (1935) 302.

[23] L. M. Lubin, and A. Sandage, The Tolman Surface Brightness Test for the Reality of the Expansion. IV. A Measurement of the Tolman Signal and the Luminosity Evolution of Early-Type Galaxies, Astron. J. 122 (2001) 108.

[24] E. J. Lerner, Evidence for a Non-Expanding Universe: Surface Brightness Data From HUDF, in First Crisis in Cosmology Conference (AIP Conf. Proc. 822), E. J. Lerner, J. B. Almeida, Eds., AIP, p. 60 (2006).

[25] E. J. Lerner, R. Falomo and R. Scarpa, UV surface brightness of galaxies from the local Universe to $z \sim 5$, Int. J. Mod. Phys. D 23 (2014) id. 1450058.

[26] V. K. Kapahi, The angular size-redshift relation as a cosmological tool, in Observational Cosmology (IAU Symp. 124), A. Hewitt, G. Burbidge, and L. Z. Fang, Eds., Reidel, Dordrecht, p. 251 (1987).

[27] M. López-Corredoira, Alcock-Paczyński cosmological test, Astrophys. J. 781 (2014) 96.

[28] M. López-Corredoira and F. Melia. Alcock-Paczyński cosmological test with BAO peak, in preparation. 